\title{
Thermal Design and Analysis for the Cryogenic MIDAS Experiment
}

Ruth McElroy Amundsen NASA Langley Research Center

\section{ABSTRACT}

The Materials In Devices As Superconductors (MIDAS) spaceflight experiment is a NASA payload which launched in September 1996 on the Shuttle, and was transferred to the Mir Space Station for several months of operation. MIDAS was developed and built at NASA Langley Research Center (LaRC). The primary objective of the experiment was to determine the effects of microgravity and spaceflight on the electrical properties of high-temperature superconductive (HTS) materials. The thermal challenge on MIDAS was to maintain the superconductive specimens at or below $80 \mathrm{~K}$ for the entire operation of the experiment, including all ground testing and 90 days of spaceflight operation. Cooling was provided by a small tactical cryocooler. The superconductive specimens and the coldfinger of the cryocooler were mounted in a vacuum chamber, with vacuum levels maintained by an ion pump. The entire experiment was mounted for operation in a stowage locker inside Mir, with the only heat dissipation capability provided by a cooling fan exhausting to the habitable compartment. The thermal environment on Mir can potentially vary over the range 5 to $40^{\circ} \mathrm{C}$; this was the range used in testing, and this wide range adds to the difficulty in managing the power dissipated from the experiment's active components.

Many issues in the thermal design are discussed, including: thermal isolation methods for the cryogenic samples; design for cooling to cryogenic temperatures; cryogenic epoxy bonds; management of ambient temperature components' self-heating; and fan cooling of the enclosed locker. Results of the design are also considered, including the thermal gradients across the HTS samples and cryogenic thermal strap, electronics and thermal sensor cryogenic performance, and differences between ground and flight performance. Modeling was performed in both SINDA-85 and MSC/PATRAN (with direct geometry import from the $C A D$ design tool Pro/Engineer). Advantages of both types of models are discussed. Correlation of several models to ground testing and flight data (where available) is presented. Both SINDA and PATRAN models predicted the actual thermal performance of the experiment well, even without post-flight correlation adjustments of the models.

\section{INTRODUCTION}

The Materials In Devices As Superconductors (MIDAS) experiment is an autonomous locker-contained payload that was launched on the Shuttle and transported to the Priroda module on the Mir space station. After 90 days of operation, it was returned to Earth via the Shuttle. The primary objective of the experiment was to determine the effects of microgravity and spaceflight on the electrical properties of hightemperature superconductive (HTS) materials. These ceramic compounds exhibit unique characteristics that could enhance and improve space-borne scientific instruments. A secondary objective of the experiment was to use commercial components for a flight experiment and evaluate their performance.

The HTS materials used were $\mathrm{YBa}_{2} \mathrm{Cu}_{3} \mathrm{O}_{7-x}$ and $\mathrm{Bi}_{2} \mathrm{Sr}_{2} \mathrm{Ca}_{2} \mathrm{Cu}_{3} \mathrm{O}_{x}$, which have transition temperatures (the point at which they become superconductive) of 86 and $99 \mathrm{~K}$, respectively. Transition temperatures can be higher than the rated value depending on the sample purity and preparation. The experiment was designed to maintain the HTS samples below $80 \mathrm{~K}$ for three separate cycles of 28 days. The experiment involved several thermal challenges. The primary one was the requirement to maintain the samples below $80 \mathrm{~K}$. This involved holding the samples under vacuum to eliminate air conduction, providing a radiation barrier, and limiting the thermal conduction of the structural supports and electrical connections. A small tactical cryocooler was used to provide cooling, and the heat generated by the unit had to be dissipated to keep it from overheating. Since the experiment was contained and operated in a locker, all heat from the experiment had to be controlled within the Mir and Shuttle requirements. 
The experiment was thermally modeled using both MSC/PATRAN (versions 5.0 and 6.0) [1] and SINDA-85 [2] software. Steady-state temperature and transient predictions were made, and were compared to both ground testing and flight performance.

\section{INSTRUMENT DESCRIPTION}

The MIDAS experiment consists of the vacuum chamber with HTS materials and their mechanical support, the tactical cryocooler and its control electronics, data acquisition and control electronics, a battery, an ion pump, a fan, and the mechanical support structure. MIDAS is shown in Figure 1 with the exterior foam and outer cover removed.

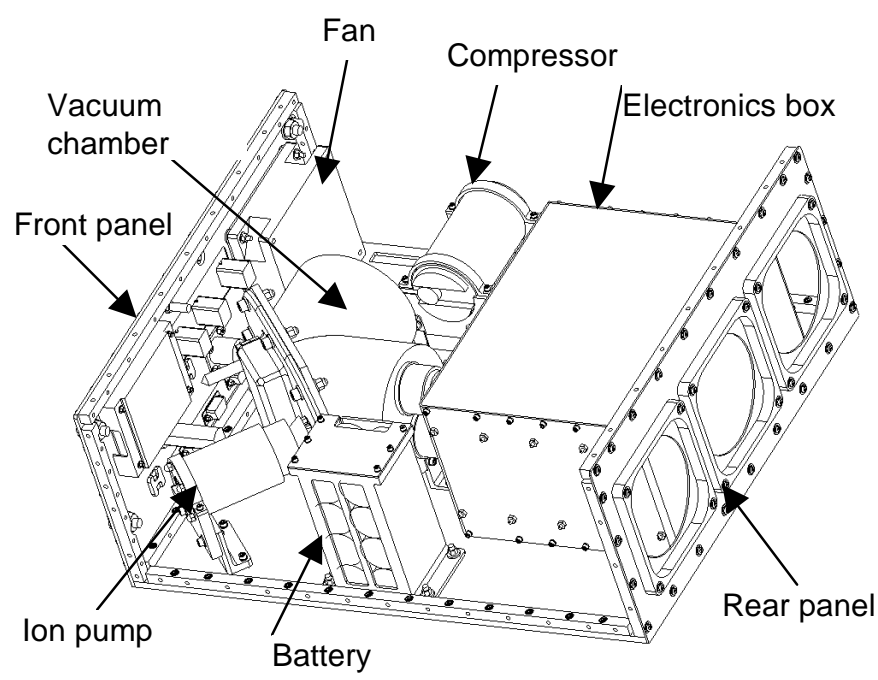

Figure 1. MIDAS Experiment Layout

Central to the MIDAS experiment are the HTS samples, which are contained on four thin ceramic substrate boards. Two boards were LaRC-fabricated, one board was supplied by a Russian co-investigator, and one was fabricated by the Eaton Corporation. The $\mathrm{LaRC}$ boards consist of $\mathrm{YBa}_{2} \mathrm{Cu}_{3} \mathrm{O}_{7-x}$ superconductive elements deposited on a polycrystalline yttria-stabilized zirconia (YSZ) substrate. Each test circuit (or board) possesses six superconductive elements, which are the dark traces shown in Figure 2. The boards are $2.5 \times 2.5$ $\mathrm{cm}$ and are mounted on the faces of a thermally conductive, hollow copper cube. Multiplexing and amplification of voltages measured from the superconductive elements and platinum resistance temperature devices are performed directly on the test circuit boards using multilayer thick film printing and active die-form multiplexers and amplifiers, resulting in active, micro-electronic eight-channel hybrid superconductive/conventional circuits. The substrates are bonded to the cube with a thin layer of epoxy. The cube cannot be supported directly on the cryocooler expander (coldfinger) because this would place excessive loads on the expander during launch. Thus, a thermally isolative support stand was designed. The flight and prototype support stands were different, as explained below in the thermal design section. The prototype was an epoxy resin / glass fiber cryogenic composite thin-walled tapered circular cone, and the flight stand was a four-legged stand of titanium alloy (Ti-6Al-4V). Both stands were designed such that the thermal conductance to the cube was as low as possible. The copper cube and support stand were radiatively insulated using a multi-layer insulation (MLI) blanket.

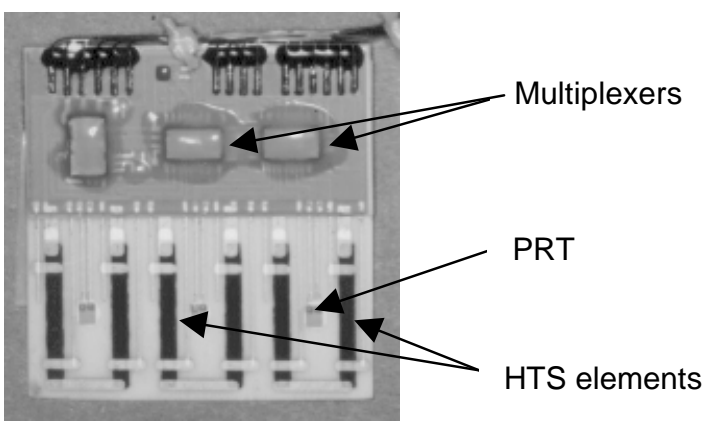

Figure 2. HTS Sample Board

The vacuum chamber is a stainless steel housing with an internal volume of approximately 1.5 liters. The chamber provides a vacuum to facilitate cooling the HTS samples; the maximum pressure level is $10^{-3}$ torr, driven by having to limit air conduction. The cryocooler expander, ion pump, and electrical connectors (to provide exit for the HTS sample power and data lines) are mounted in the walls of the vacuum chamber. Once the chamber is pumped down, an ion pump mounted on the vacuum chamber via a miniconflat style flange maintains the vacuum. The ion pump is an integrated unit; the ion pump and the control electronics are in the same package. The ion pump has a pumping speed of $0.5 \mathrm{l} / \mathrm{s}$ at $10^{-5}$ to $10^{-8}$ torr, a maximum power usage of 3 watts over its operating range of 10 to 14 VDC, and a minimum operating life of 9000 hours. The ion pump is powered via a DC to DC converter when the MIDAS experiment is powered and a 12 VDC backup battery when the experiment is unpowered.

The Texas Instruments tactical linear cryocooler used can provide 1 watt of cooling to $80 \mathrm{~K}$ with an ambient temperature of $20^{\circ} \mathrm{C}$. The cryocooler uses 85 $\mathrm{cc}$ of helium in a sealed canister at a pressure of $250 \mathrm{psi}$ as a working fluid and has a lifetime of approximately 4000 hours. The cryocooler compressor, which dissipates about $35 \mathrm{~W}$, is attached to the experiment baseplate with aluminum brackets. The cryocooler expander, which dissipates about $15 \mathrm{~W}$, is mounted to a face of the vacuum chamber via an O-ring sealed flange. The coldfinger extends from the expander into the vacuum chamber through an opening in the wall. The cryocooler compressor and expander are connected via a small diameter helium transfer line.

The remainder of the experiment includes the electronics, battery, and a fan in the front panel. The data acquisition and control electronics consist of nine printed circuit boards in a ruggedized card cage. With 
the exception of two boards built by LaRC, all other boards are commercially provided. The battery pack serves as a 12 VDC backup supply for the ion pump when the MIDAS experiment is disconnected from external power. The fan is a small DC muffin fan that uses a maximum of $3.4 \mathrm{~W}$ over its operating range of 24 to 56 VDC. The fan is attached to the MIDAS experiment front panel and provides approximately 65 $\mathrm{cfm}$ of airflow through the experiment housing.

The MIDAS experiment mass is approximately $24 \mathrm{~kg}$. Except for the fan (attached to the cover), all experiment hardware is fastened to an aluminum baseplate. The experiment is contained in a $40 \times 47 \times 19$ $\mathrm{cm}$ aluminum cover that is attached to the baseplate. There are three fan exhaust holes in the rear panel of the housing. The exterior of the experiment is covered by closed-cell foam required by both Shuttle and Mir. Openings in the foam are provided for the front panel controls and fan screen intake and exhaust ports. The experiment mounts into the Shuttle mid-deck lockers and the Mir single-stowage lockers by simply sliding into place and closing the locker front face - there are no hard mount points. This limits the thermal transfer out of the experiment via direct conduction, and forces the heat exchange to depend mainly on efficient airflow. On both Shuttle and Mir, power was provided via a cable connection to the front panel. The experiment was operated only on Mir; power on the Shuttle was provided for ion pump operation only. Once on Mir, astronauts activated the experiment using circuit breakers on the front panel.

\section{THERMAL DESIGN}

The challenges in the MIDAS thermal design arose out of the need to maintain certain components at cryogenic temperatures and others at room temperature. The challenges were compounded by the fact that MIDAS was autonomous and enclosed in a relatively small locker, with a limited amount of power. Issues that had to be addressed included materials and electronics selection for cryogenic use, optimization of the structural design and thermal radiation protection for the cryogenic portion, and heat exchange with Priroda using forced fan cooling.

One important thermal issue was the bonding of the ceramic boards to the copper cube. This bond had to withstand multiple cycles from room temperature (or the $75^{\circ} \mathrm{C}$ cure temperature) to $75 \mathrm{~K}$. Many epoxies were tested, and most proved too brittle, shattering the ceramic boards when they were cooled. Other requirements on the epoxy were that it be thermally conductive, both to facilitate spreading the heat from the active electronic devices on the boards, and to minimize the temperature differential between the coldfinger and the samples. Also, the epoxy had to have very low outgassing characteristics, to minimize both the outgassing load within the chamber and the contamination of the HTS materials. The EA9309 epoxy selected had a $75^{\circ} \mathrm{C}$ cure required to shorten the cure time, but even from this initial temperature it successfully withstood dozens of cycles to cryogenic temperature without excessive stress on the boards. One interesting discovery was that stress on the boards was intimately related to their exact mounting configuration. If the boards were mounted on the cube such that two edges were in contact, the shrinkage created by taking the assembly down to cryogenic temperatures caused the edges of the boards to push on each other and stressed the boards to failure at the edges. Thus, testing the epoxy by bonding substrates to flat copper test pieces was not sufficient to ensure a successful bond. The bond used was kept to a thickness of roughly $0.005 \mathrm{~cm}$ by using small glass beads mixed into the epoxy. The thin bondline worked to minimize the volatiles released, minimize the thermal resistance, and mitigate the stresses produced.

Another thermal issue was the support of the HTS sample cube. The coldfinger of the expander had a limit on the load it could withstand, so the decision was made to support the cube and samples directly, and connect them to the coldfinger via a thermal strap. The support was initially designed as a thin-walled fiberglass tapered circular cone, with the flange and wall thicknesses of the cone optimized for the minimum thermal conductance while providing adequate structural support. Two problems were eventually found with this cone. One was that it continued to outgas, and was in danger of losing structural integrity as well as producing a substantial vapor load. Also, since it was a solid piece, an MLI blanket had to be placed under the foot of the cone to provide radiation protection. This required the use of two separate MLI blankets that were difficult to assemble in the limited vacuum chamber space and provided an excessive surface area, which slowed bakeout and vacuum pumpdown. The support was redesigned as a four-legged titanium alloy (Ti-6Al-4V) stand, with each leg having a $1.2 \times 1.2 \mathrm{~mm}$ square cross section. This allowed an MLI blanket to be wrapped through the legs of the stand, around the cube, thermal strap, and coldfinger tip, yielding a smaller single blanket. The titanium plate under the cube used four raised pads at the mounting points to decrease the contact area and increase thermal resistance. The redesigned stand used for flight is shown in Figure 3.

A thermal strap was used between the copper cube and the coldfinger to allow cooling of the HTS samples. This strap needed to provide maximum thermal transport without being a rigid structural element that would transmit loads from the cube to the coldfinger. Several designs were evaluated; the one actually used consisted of 11 strips of $0.1-\mathrm{mm}$ thick copper foil. The strips were $2.5 \mathrm{~cm}$ wide by $7.6 \mathrm{~cm}$ long, and were press-mounted on both ends. Indium foil was used to minimize the contact resistances at the mounting points. The distance between the contact points at the coldfinger and cube 
was only $1 \mathrm{~cm}$. In both ground testing and in flight, the strap performed well, resulting in a thermal gradient of about $2^{\circ} \mathrm{C}$ between the HTS samples and the coldfinger.

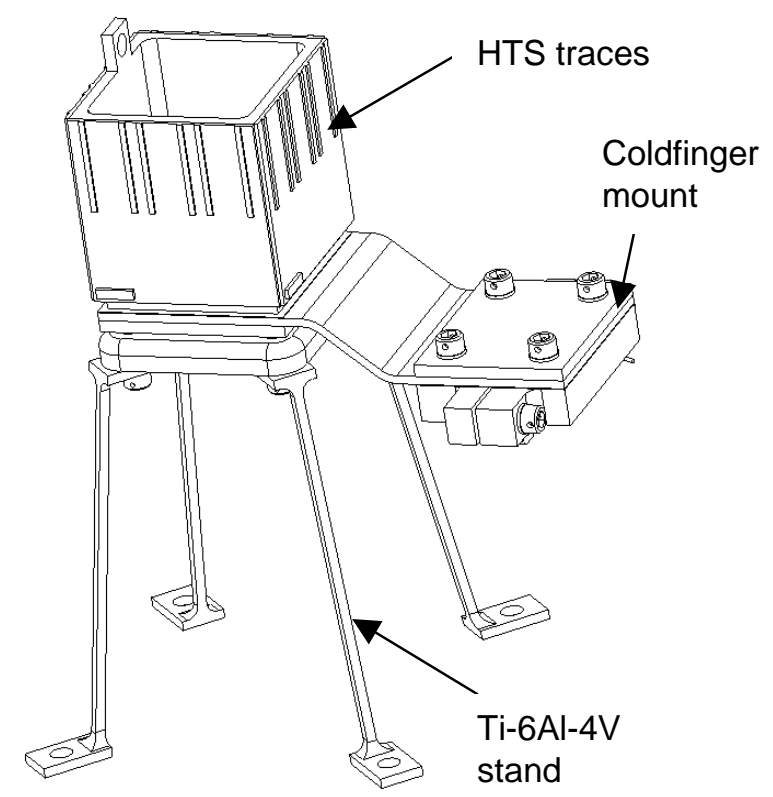

Figure 3. Cryogenic Support Stand

Thin-film platinum resistance thermal sensors (PRTs) were used on the cube and coldfinger for temperature measurement. On each board there were three PRTs mounted in between the HTS samples, and one mounted near the active electronics. Although neither the thermal sensors nor the active die-form electronic components are rated for operation at $75 \mathrm{~K}$, all performed well. The die-form electronics were prescreened for cryogenic operation; once a component was found to perform successfully at the low temperatures, it could be used with confidence. The lower temperature limit on operation of the electronics was about $72 \mathrm{~K}$; below this temperature the operation of the electronics was not reliable. This placed a narrow window on the optimum operation of the cooler: to control between 72 and $75 \mathrm{~K}$. The power dissipation of the PRTs was minimal. The power contribution from the active electronics was minimized by staggering the measurement cycles. In a 30-minute period, each board was measured once, with a seven minute pause between boards. The die were connected to the electrical traces on the boards with extremely thin gold wire bonds. These wire bonds were remarkably fragile, and had to be protected. The method chosen was to encapsulate the die with a layer of epoxy. As with the bond of the boards to the cube, several epoxies were evaluated for this use, with most causing the die to shatter or separate from the board. The EA9309 epoxy was eventually selected for this application as well.

The two remaining sources of heat to the boards, in addition to the support stand and active electronics already discussed, were radiation through the MLI blanket and conduction along the wires used for power and data on the boards. The MLI blanket design was limited by the fact that there was less than $2 \mathrm{~cm}$ between the cube and the vacuum chamber wall in some places. Another limit was imposed by the desire to limit surface area that would contribute an offgassing load on the ion pump. The flight blanket consisted of four layers of perforated Mylar (0.02-mm thick), aluminized on both sides, interleaved with Dacron netting, and outer layers of $0.07-\mathrm{mm}$ thick Kapton to facilitate handling. The thermally preferred alternative would have been for both the inside and outside of the blanket to be aluminized, but due to the danger of shorting the electronics on the HTS boards, Kapton was used as an insulator for the innermost layer. Slits were cut in the blanket to facilitate venting and pumpdown. The number of wires exiting the boards was minimized by the use of active electronics on the boards for multiplexing. However, there were still 72 wires passing from the cryogenic samples out to the ambient temperature chamber walls. The heat load this imposed was minimized by the use of 36-gauge manganin wires with thin Formvar insulation.

Thermal issues outside the vacuum chamber related to the dissipation of component power. The power used by each component is shown in Table 1. Under normal operation, a total of $55 \mathrm{~W}$ had to be removed from the experiment, while maintaining the components within their operating temperature ranges. The maximum allowable temperature of the front panel, as defined by Mir and Shuttle safety documentation, was $49^{\circ} \mathrm{C}[3,4]$. The maximum environmental temperature that could be experienced within Priroda was $40^{\circ} \mathrm{C}$, although temperatures actually experienced during flight were normally near $25^{\circ} \mathrm{C}$. The heat was removed using a single fan. The requirement for operation on the Priroda module of Mir was that all air had to be taken in from the front panel, and exhausted from the rear of the experiment. Although the experiment was not designed for operation on the Shuttle, the experiment had to be thermally fail-safe to allow acceptable temperatures in the case of inadvertent activation while still on-board the Shuttle in a closed-back locker.

Table 1. Component Dissipated Power

\begin{tabular}{|l|c|c|}
\hline Component & $\begin{array}{c}\text { Maximum } \\
\text { (W) }\end{array}$ & $\begin{array}{c}\text { Typical } \\
\text { (W) }\end{array}$ \\
\hline Data Electronics & 19 & 16 \\
\hline Cryocooler Compressor & 35 & 17.5 \\
\hline Cryocooler Electronics & 10 & 10 \\
\hline Cryocooler Expander & 15 & 7.5 \\
\hline Fan & 3 & 3 \\
\hline lon Pump & 3 & 1 \\
\hline Total Power & 80 & 55 \\
\hline
\end{tabular}

The fan was sized to be able to move at least 65 cfm of air at the lowest supply voltage that could be experienced on Mir. The front panel opening size was set by the fan size. Screens were required because of 
the possibility of air-borne particulates on Mir, and were chosen for minimum airflow obstruction. A spreadsheet was used to determine the optimum outlet area for the rear panel exhaust ports. The equations used are shown in equation [1] below. The calculation produces the curve of air flow rate versus outlet area shown in Figure 4. This curve was used to determine that any total outlet area for fan exhaust above $160 \mathrm{~cm}^{2}\left(25 \mathrm{in}^{2}\right)$ would be acceptable. The spreadsheet used to calculate this was a modification of the process used for fan calculations on the LASE project, and can be found at the WWW address $h$ ttp://ixeab3.larc.nasa.gov/analtool.html.

$$
\begin{aligned}
& \dot{Q}=\dot{m} C_{p} \Delta T=\rho \dot{V} C_{p} \Delta T \\
& \dot{m}=C_{D} A P \sqrt{\frac{2 g \gamma}{R T(\gamma-1)}\left[\left(\frac{P_{2}}{P_{1}}\right)^{\frac{2}{\gamma}}-\left(\frac{P_{2}}{P_{1}}\right)^{\frac{\gamma+1}{\gamma}}\right]}
\end{aligned}
$$

where $Q$ is the heat flow rate, $m$ is the mass flow rate, $\mathrm{C}_{\mathrm{p}}$ is the specific heat of air, $\Delta T$ is temperature change, $\rho$ is air density, $\dot{V}$ is air volume flow rate, $\mathrm{R}$ is the gas constant, $A$ is cross-sectional area, $g$ is gravitational acceleration, $\gamma$ is the ratio of specific heats, $C_{D}$ is the drag coefficient, and $\mathrm{P}_{1}$ and $\mathrm{P}_{2}$ are the pressures at the fan intake and exhaust.

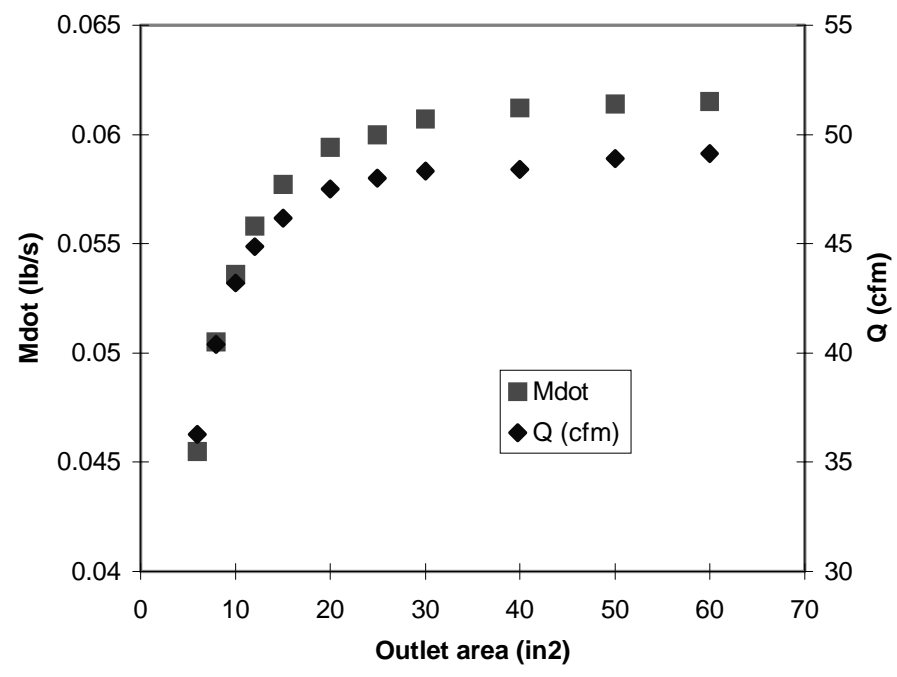

Figure 4. Fan Performance versus Outlet Area

The two main components of the cryocooler, the compressor and expander, had to be kept as cool as possible for efficient operation of the cryocooler. Their efficiencies decrease at higher temperatures -- the absolute upper limit for MIDAS was $86^{\circ} \mathrm{C}$. The compressor was mounted using aluminum brackets, and placed directly in the path of the fan airflow. The expander generates less heat, but its temperature is more critical in determining the efficiency of operation of the cryocooler. The portion of the expander where the heat was dissipated was on the exterior of the vacuum chamber. Since this was a small part, the surface area needed to be increased to facilitate cooling. A finned flange was used on the outer diameter of the expander, and the expander was placed such that it was the first item in the line of the fan airflow, and thus received the coolest possible air. The flange was attached to the expander with epoxy, thus increasing the thermal transfer, but making it difficult to remove.

In general, the ground testing could be assumed to be a fairly good representation of on-orbit behavior, since cooling used forced airflow and was not dependent on natural convection. The only area for which that did not hold true was within the electronics box. The heating here was mainly from the CPU chip itself, although all components were chosen for their low power output. Since the electronics box was a closed unit, the interior was dependent on air conduction and contact conduction for removal of heat. On Earth, the transfer of heat from the components to the box exterior would be aided by natural convection, which would not be the case on-orbit. Thus it was desirable to be able to demonstrate a good safety margin in the electronics box temperature during ground testing. Originally, the CPU chip was designed to have a copper strap connecting it to the box exterior, so that its temperature would be kept very close to that of the aluminum box. However, this strap caused electrical grounding problems during ground testing, and was eventually removed from the flight unit.

\section{THERMAL MODELING}

Thermal modeling of the experiment was done in both PATRAN and SINDA-85. Since the design of the experiment was done in the CAD software Pro/Engineer, parts could easily be imported to PATRAN for meshing and analysis. Also, since the structural analysis was performed using PATRAN models, the models could be shared between the structural and thermal analysts, resulting in substantial increases in efficiency and accuracy. Certain parts were easier to model in PATRAN, as well as allowing easier detailed modeling since Pro/Engineer geometry was available. In general, the modeling in SINDA allows easier transient plotting and quick "what-if" analyses such as changes in MLI effective emissivity, but limits the model to relatively large lumped nodes. The PATRAN models allow detailed evaluation of heat flow, temperature gradient, changes in materials and overall thermal visualization. The $P /$ Thermal analysis engine was used in all runs of the PATRAN models. The numbers of nodes in each of the models are shown in Table 2. 
Table 2. Thermal Model Sizes

\begin{tabular}{|l|c|}
\hline Model & Number of nodes \\
\hline PATRAN models & \\
\hline Vacuum chamber & 2300 \\
\hline Electronics box & 3400 \\
\hline Cube/stand & 2800 \\
\hline Full experiment & 6400 \\
\hline SINDA-85 models & \\
\hline Cube/HTS & 40 \\
\hline Full experiment & 16 \\
\hline
\end{tabular}

All models used temperature dependent material properties. The SINDA model of cryocooler operation used a bivariate array for the cryocooler performance, with load capability dependent on both ambient temperature and coldfinger temperature. The effective emissivity $\left(\varepsilon^{*}\right)$ of the MLI in the SINDA model was a temperature dependent array derived from tested values, with an average value of about 0.05 [5]. In general, the cryogenic portion of the experiment was modeled separately from the ambient temperature portions. This simplified modeling and results visualization. Since the cryogenic operation had little effect on other component temperatures, except through the level of cryocooler power used, this approach was satisfactory.

One of the first models developed in PATRAN was that of the vacuum chamber and cryocooler expander. The fine-meshed PATRAN model allowed a detailed look at the heating around the cryocooler expander. Several parametric cases were run to evaluate the effectiveness of the forced air cooling, the effect of changing the fin area and the amount of heat transferred through the vacuum chamber and its aluminum mounting foot. The final fin configuration increased the external area of the expander by a factor of 28. This allowed the expander to remain below $60^{\circ} \mathrm{C}$ even in the worst case. The majority of the cooling was provided by convection to the forced air; the stainless steel walls of the vacuum chamber did not provide a good conduction path for heat flow.

The electronics box was also modeled in PATRAN, which allowed the heating of the individual chips to be evaluated. The largest heat producer was the 486 microprocessor chip. Conduction along the PC boards, contact resistance at the mounting points, and air conduction within the box were difficult factors to estimate. As mentioned, a thermal strap was originally planned to connect the chip to the box cover, but was later eliminated. The detailed model gave a good indication of how rapidly the powered components would self-heat, how much of their heat would be transferred to the box, and where the heat was being allowed to escape.

A PATRAN model of the entire experiment was created using the detailed models of the vacuum chamber and electronics box, and adding fairly simple parts for the battery, experiment baseplate, cryocooler compressor and cryocooler electronics. This model did not include the cryogenic parts within the vacuum chamber, but allowed a detailed look at heating of all components, as well as an evaluation of which components had the greatest effect on heating of the front panel. An airflow conductor was used to model the air exchange with Mir; Mir temperature was assumed to be stable. All contacting parts had contact conductances assigned, except where it was more conservative to assume perfect contact. The self-heating of the components was fairly well-controlled, and none were predicted to exceed their maximum operating temperatures, even using worst-case power assumptions. A thermal map of predicted nominal operation is shown in Figure 5. The temperature requirement on the front panel, to keep it under $49^{\circ} \mathrm{C}$, was the most difficult to achieve, since the maximum potential environmental temperature was $40^{\circ} \mathrm{C}$. In fact, with the worst-case assumption required by NASA regulations, that the fan could stall out in a locked rotor condition (resulting in a dissipated heat of $6.5 \mathrm{~W}$ ), the front panel temperature was predicted to reach the Shuttle-imposed limit temperature.

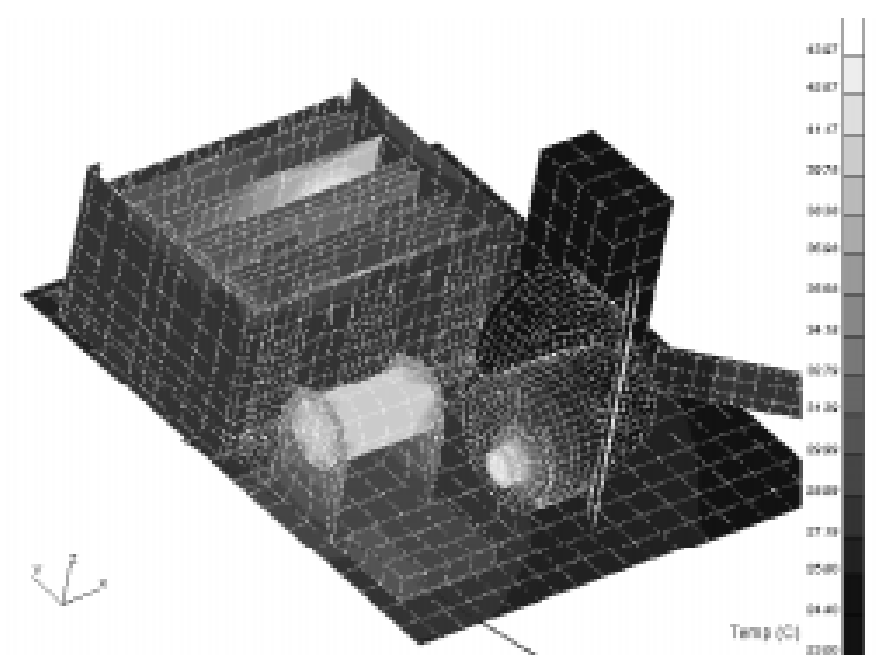

Figure 5. PATRAN Model Prediction

A detailed PATRAN model of the cube with the boards and active electronics, shown in Figure 6, was generated in order to evaluate whether the active electronic parts would induce unacceptable gradients on the HTS boards. In flight, this gradient was observed to be fairly small - the delta between a sample PRT and the PRT near the electronics was about $1.4^{\circ} \mathrm{C}$. This is very close to the model prediction shown. The gradients across the sample PRTs were very small in flight - less than the noise in the PRT measurements, also as predicted. 


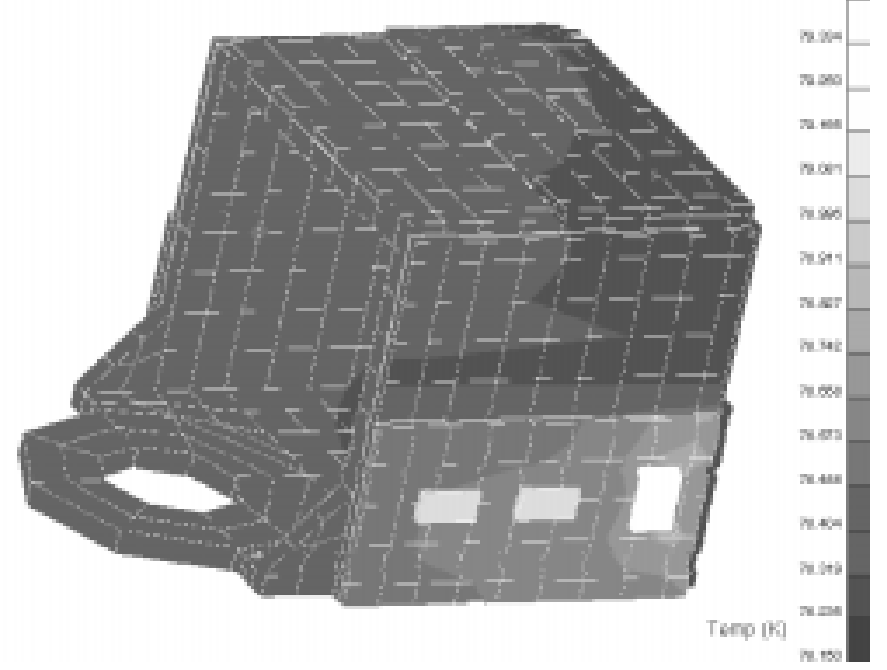

Figure 6. PATRAN Cube/HTS Model Prediction

After the redesign of the cryogenic support stand for flight, a rapid analysis was necessary to evaluate whether the thermal performance would be satisfactory. A PATRAN model of the cube, coldfinger mount and support stand was created directly from the Pro/Engineer assembly file. This model, shown in Figure 7, was meshed using tetrahedral elements, so that the parts would not have to be re-created as PATRAN solids. This type of modeling allowed extremely rapid model generation and analysis to evaluate the design of the titanium alloy stand for cryogenic use. This analysis was performed from start to final results in 30 minutes, and showed that the support stand performance would be acceptable.

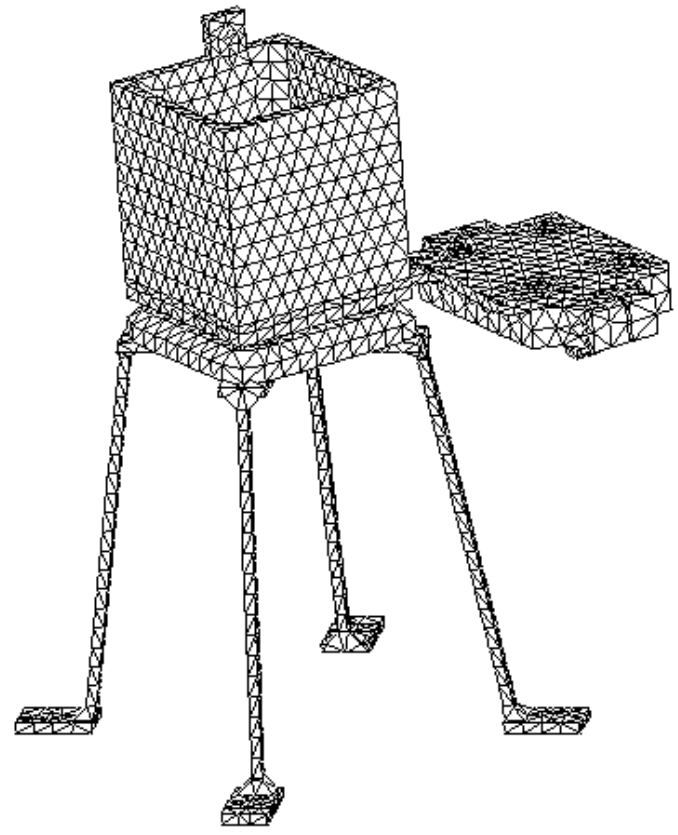

Figure 7. PATRAN Cube/Stand Model

A SINDA-85 model of the entire experiment (not including the cryogenic portion) was created, and correlated to the values predicted by the PATRAN model, to allow quick analyses, as well as changes via text editing. A SINDA-85 model of the cryogenic portion (cube, coldfinger and HTS samples) was created to facilitate evaluation of transients, design changes, and the heating distribution (e.g., radiation versus wire conduction). An example heating distribution during cooldown is shown in Figure 8, with the heat load divided between radiation, wire conduction, support mount conduction, and heating from the active components on the cryogenic boards. The total heat load absorbed by the cryocooler was predicted to be $410 \mathrm{~mW}$ during nominal cooldown and $475 \mathrm{~mW}$ in the worst case. Once the cooler reached steady state at $75 \mathrm{~K}$, the measurement frequency decreased and the total load was 330-390 mW depending on the case. These loads are well within the cooler capacity of $1 \mathrm{~W}$.

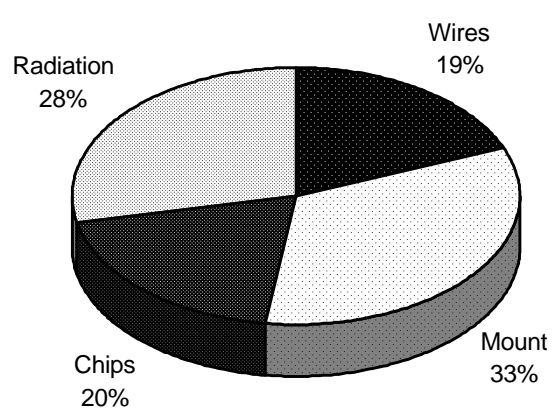

Figure 8. Heat Load Distribution during Cooldown GROUND TESTING AND FLIGHT PERFORMANCE

The SINDA-85 model of the experiment's cryogenic portion was compared to the ground testing data, with no post-test correlation or adjustment of the model. This plot of the coldfinger temperature (for prediction versus test) is shown in Figure 9. The analysis predicts the shape of the cooldown very well, and is conservative as it reaches the steady-state condition. The cryocooler successfully cooled the entire sample mass to less than $80 \mathrm{~K}$ in less than an hour. The model was not compared to the flight data because there was an anomaly in the flight cooldown that has not yet been explained.

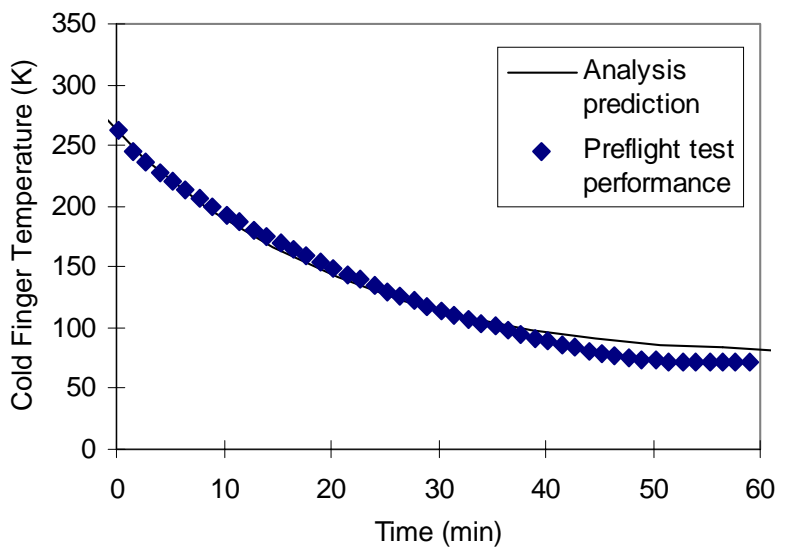

Figure 9. Prediction vs. Performance for Cooldown 
Both the PATRAN and SINDA models are compared to the actual performance of the flight experiment in terms of self-heating of ambient temperature components. The four points that can be compared are the locations where housekeeping temperature sensors were mounted - the CPU chip, cryocooler compressor, cryocooler expander and vacuum chamber. Plots comparing the behavior of the first three are shown in Figures 10-12 (the vacuum chamber simply tracked ambient at about $25^{\circ} \mathrm{C}$ and thus is not shown). These models have not been altered to correlate with the actual performance except that the power for the cryocooler was adjusted to reflect the actual operating environment. Both ground test data and flight data are shown. Ground tests differed from the flight operation in that the ground testing compressed the 90-day flight experiment cycle into four days, and thus measurements were taken much more frequently.

The comparison of the behavior of the CPU chip temperature in ground testing, flight performance, and both the SINDA and PATRAN modeling is shown in Figure 10. As expected, the CPU was warmer during flight than during ground testing, since during flight there was no cooling due to natural convection. Also, the SINDA model grossly underpredicts the CPU temperature, which was expected since the SINDA model lumps the entire 486 board as a single node and thus averages its temperature. The reason that the PATRAN model prediction is too low has not been determined - it could be that the CPU chip actually dissipated more power than specified, or that the contact resistances were underestimated.

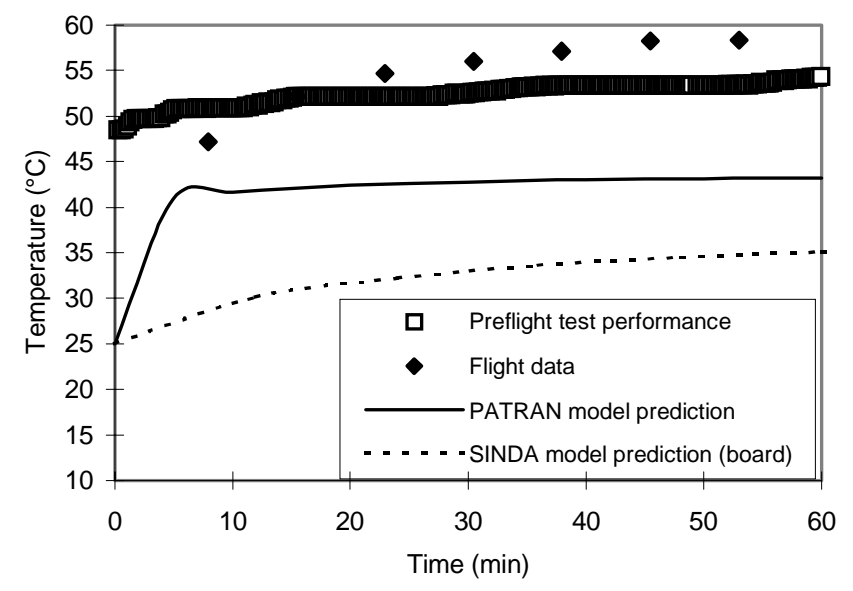

Figure 10. Prediction vs. Performance for CPU

A similar plot for the cryocooler compressor is shown in Figure 11. In this case, the preflight ground test produces higher temperatures than flight. This is because the ground test used more frequent HTS sample measurements than flight, and thus there was a higher dissipated load on the cube and a higher power in the compressor was necessary. The SINDA model has a very close correlation to the flight condition; the
PATRAN model prediction is slightly low, but is within $2^{\circ} \mathrm{C}$.

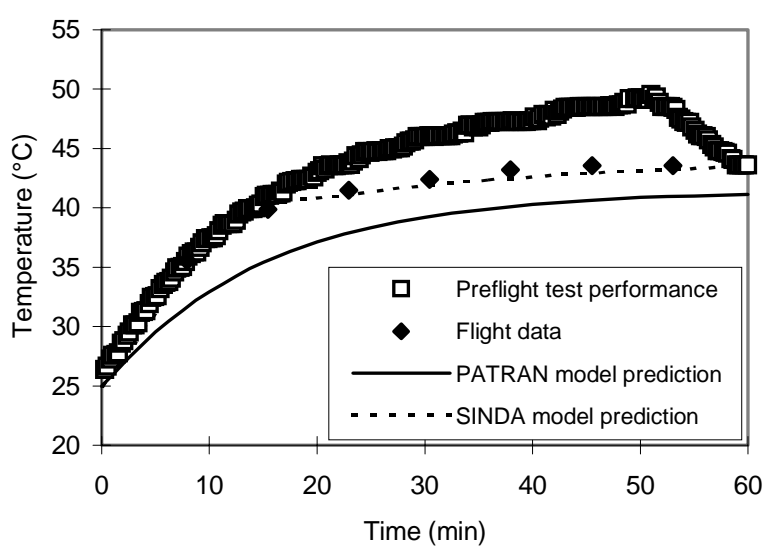

Figure 11. Prediction vs. Performance for Compressor

The plot for expander temperatures is shown in Figure 12. Again, the ground test shows a higher temperature than flight due to the higher measurement frequency during ground tests. The PATRAN model shows a very close correlation to the flight data -- less than a $1^{\circ} \mathrm{C}$ difference. The SINDA model prediction is low by about $5^{\circ} \mathrm{C}$, and also has a slower response time, probably due to the fact that the model of the expander is a single lumped node.

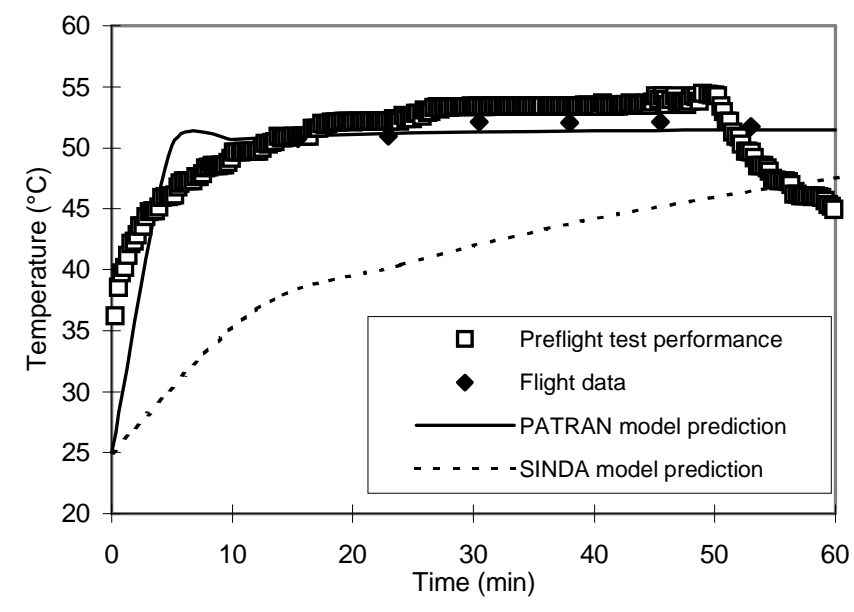

Figure 12. Prediction vs. Performance for Expander

These models can be adjusted to more closely predict the actual performance. However, it is valuable to examine the accuracy of the models when no correlation has been done, since a model is often used for design decisions before it has been correlated. The PATRAN model predicts the tested thermal behavior quite well in most cases, both in terms of steady-state temperatures and transient curves. The only exception was the CPU chip temperature, which was off by $15^{\circ} \mathrm{C}$. This could be a result of insufficient detail in the board model to be able to predict a chip temperature, i.e., not including solder joints, detailed trace paths, connector pins, etc. The SINDA model shows some of the 
disadvantages of relatively large lumped nodes in that it does not always predict the maximum temperature or transient rate well. The SINDA model of the cryogenic portion predicted the cooldown extremely well. The PATRAN model cryogenic cooldown prediction is not shown, since it was not fully updated to the flight configuration. However, it predicted the same basic behavior accurately for the prototype support stand and cube.

\section{CONCLUSIONS}

The thermal design of MIDAS was successful in allowing cooling of the samples, and in maintaining the other components within their operating temperatures. Through extensive testing an epoxy was selected and bonding process developed to bond the sample ceramic boards to the copper cube support for successfully withstanding multiple thermal cycles from $75 \mathrm{C}$ to $75 \mathrm{~K}$. A unique support stand was designed to thermally isolate the cryogenic HTS samples and also minimize outgassing within the vacuum chamber. Radiation shielding and wiring design for the cryogenic allowed successful cooling. The calculated fan exhaust area and fin area for the expander were successful in controlling experiment self-heating using airflow from the fan. The Mir environment was consistent over the 90-day time period, with temperatures remaining at about $25^{\circ} \mathrm{C}$.

The SINDA and PATRAN models of the experiment both provided valuable insights. Both compare well with the experiment performance, even without correlating adjustments. PATRAN predictions were within $2^{\circ} \mathrm{C}$, and SINDA predictions were within $5^{\circ} \mathrm{C}$, except for the CPU chip temperature which was off by $15^{\circ} \mathrm{C}$ in the PATRAN model and $25^{\circ} \mathrm{C}$ in the SINDA model. This may illustrate the dangers of predicting a computer chip temperature without a fully detailed partlevel model of the electronics board. The PATRAN model gradient predictions for the thermal strap and cube were validated in ground testing and flight. The predicted load on the cryocooler was found to be accurate in that the cooler performed as expected. In general, the SINDA modeling proved more valuable for quick analysis of transients and simple 'what-if' cases. The PATRAN model was more useful for detailed gradient analysis, correlation to test data, and determination of stresses via translation to structural analysis.

\section{ACKNOWLEDGMENTS}

The work of the entire MIDAS team at NASA Langley Research Center is gratefully acknowledged, including the assistance of Dr. Stephanie Wise, Matt Hooker, Jim Wells, Rick Hopson and Eli Siman-Tov in supplying and formatting data. Particular thanks go to Jill Marlowe for sharing of analysis models and Robert Dillman for creation and sharing of CAD models and graphics. The assistance of Brian Killough and Bill
Alexander with the convection equations is acknowledged with thanks.

\section{REFERENCES}

1. MSC/PATRAN User Manual, MacNeal-Schwendler Corporation, Version 5.0 (March 1996) and 6.0 (August 1996).

2. COSMIC: SINDA '85/FLUINT, Systems Improved Numerical Differencing Analyzer and Fluid Integrator, Version 2.3.

3. NSTS 21000-IDD-MDK, Shuttle/Payload Interface Definition Document for Middeck Accommodations, March 1988.

4. US/R-002, Hardware General Design Standards and Test Requirements, Korolev Rocket \& Space Corporation Energia, December 1994.

5. Doenecke, "Survey and Evaluation of Multilayer Insulation Heat Transfer Measurements," SAE 932117, July 1993

\section{ADDITIONAL SOURCES}

Additional MIDAS information and photographs can be found on the MIDAS WWW home page at http://ixeab4.larc.nasa.gov/midas/midas.html.

\section{DEFINITIONS, ACRONYMS, ABBREVIATIONS}

$\begin{array}{ll}\text { cfm } & \text { cubic feet per minute } \\ \text { HTS } & \text { High-temperature Superconductors } \\ \text { LaRC } & \text { Langley Research Center } \\ \text { MIDAS } & \text { Materials In Devices As Superconductors } \\ \text { MLI } & \text { multi-layer insulation } \\ \text { LaRC } & \text { Langley Research Center } \\ \text { WWW } & \text { World Wide Web }\end{array}$

